\title{
Dyshormonogenesis Emerging as a Predominant Cause of Congenital Hypothyroidism in India - A Mini Review
}

Sudha Rathna Prabhu*

The Tamil Nadu Dr MGR Medical University , Chennai , Tamil Nadu , India

Article Info

\section{Article Notes}

Received: May 02, 2018

Accepted: July 04, 2018

\section{${ }^{*}$ Correspondence:}

Dr. Sudha Rathna Prabhu, MBBS, DNB, (PhD), Pediatrician and Research Scholar in Maternal And Child Health at Institute of Obstetrics and Gynaecology Hospital for Women \& Institute of Child Health and Hospital for Children, Egmore Chennai, Tamil Nadu, India; Affiliated to The Tamil Nadu Dr MGR Medical University, Chennai, Tamil Nadu, India;

Email: sudharathnaprabhu@gmail.com; rathinahealthcarecentre@gmail.com.

(c) 2018 Prabhu SR. This article is distributed under the terms of the Creative Commons Attribution 4.0 International License.

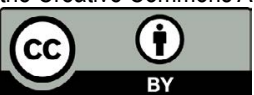

\section{Keywords}

Congenital hypothyroidism

lodine deficiency disorders

Dyshormonogenesis

Intellectual impairment

lodized salt; Goiter

Urinary iodine levels

\section{ABSTRACT}

Congenital hypothyroidism $(\mathrm{CH})$ is one of the most common preventable and treatable cause of intellectual impairment in children. Among known etiologies dysgenesis due to abnormal anatomical development and dyshormonogenesis as a result of deranged physiological functioning of fetal thyroid gland. account for at least $90 \%$ of causes of $\mathrm{CH}$. While review of literature report thyroid dysgenesis as the most common etiology of $\mathrm{CH}$ currently focus is on iodine status adequacy in women before and during pregnancy and after delivery and lactation. Worldwide reports of previously iodine sufficient countries declared presently as iodine insufficient and recent publications of several Indian states consuming lesser amounts of iodized salt seem to indicate that iodine related pathophysiological factors are emerging as predominant etiologies of $\mathrm{CH}$. The most vulnerable pregnant women and newborns are prone to be affected with iodine imbalances leading to pregnancy and perinatal related complications. Analysis of demographic, biochemical, clinical and statistical data by Indian Council of Medical Research (ICMR) in a multi-centric pilot study on congenital hypothyroidism has clearly shown that dyshormonogenesis is a leading cause of $\mathrm{CH}$ in neonates born in India. The need of the hour is to consider mandatory newborn screening for $\mathrm{CH}$ in all live newborns and further conduct country wise ethnic and culture oriented research studies with special emphasis on iodine status, genetic predispositions and lifestyle changes impact on all vulnerable populations and design effective early therapeutic strategies for management of $\mathrm{CH}$ to prevent intellectual impairment.

\section{Introduction}

Globally, malnutrition both as under and over nutrition is a major health hazard and the most vulnerable are women and children ${ }^{1}$. Among essential micronutrients iodine is vital for normal development of thyroid gland. Most of the iodine uptake takes place inside thyroid gland and hormones synthesis and functions are entirely dependent on the bioavailability of iodine in consumed diet. Risk factors causing iodine imbalances are thus prone to affect thyroid gland and initiate a cascade of biochemical changes. This leads further to a spectrum of endocrine related metabolic disorders referred to as iodine deficiency disorders (IDD) within vulnerable human biological systems and pose a constant threat to the entire life time of an individual from in utero conception stage till senility. Worldwide iodine deficiency constitute the single largest cause of preventable brain damage ${ }^{2,3}$ and most of consequences of IDD are irreversible but preventable when intervened early ${ }^{1}$. When predisposing socio demographic factors favouring malnutrition operate within vulnerable population groups, detrimental effects are inevitable and most severe health impact occurs in maternal 
and neonatal populations. Congenital hypothyroidism $(\mathrm{CH})$ is one of the most common preventable and treatable endocrine cause of mental impairment in children and is usually caused by iodine deficiency ${ }^{1-3}$ resulting in decreased and / or absence of biological actions of the thyroid gland.

\section{Risk Factors in CH}

While Western literature report dysgenesis as most common etiology of $\mathrm{CH}$, dyshormonogenesis (DH) seems commoner in Indian population. Recently published studies are reporting increase in $\mathrm{CH}$ prevalence predominantly due to dyshormonogenesis particularly in Asian population ${ }^{4-6}$. Iodine deficiency is on the rise and as per National Health and Nutrition Examination Survey (NHANES 2005-2008) survey in USA ,57\% of pregnant women had urinary iodine levels less than $150 \mathrm{mcg} / \mathrm{L}$ suggestive of iodine insufficiency ${ }^{7}$.As per recent global estimate, 1.88 billion people are at risk of iodine deficiency. 241 million children have an inadequate iodine intake and over half the children with insufficient iodine intake live in South-East Asia and Africa $^{8,9}$. Among Indian populations based studies ${ }^{1,6,8}$, there is a gradual rise in number of children with thyroid dyshormonogenesis compared to thyroid dysgenesis. Major regional risk factors attributed and observed in ICMR pilot study at Chennai centre ${ }^{10,11}$ are consanguineous marriages, practice of marriages among natives of the same district and caste groups particularly in South India. A scientific letter on dyshormonogenesis as etiology of $\mathrm{CH}$ in siblings has been reported by the author ${ }^{12}$ and this mini review is an attempt to further unfold etiopathogenesis of congenital hypothyroidism in Indian population.

Concept of "Golden Period" for intervention: The time period from conception till two years of age represents the first 1000 days of a baby and is referred to as "GOLDEN PERIOD "when fetal programming links nutritional and environmental factors during embryonic and foetal development with risk of diseases in later life ${ }^{13}$. Detection and treatment of specific diseases during this crucial period provide best of health outcomes of affected babies. In normal children exposure to time oriented stimuli will help develop their cognitive abilities. This normal phenomenon occurs because a direct link exists between early exposure and level of success of these children as they become adults. The same phenomenon applies to children who are diagnosed with congenital hypothyroidism during the same GOLDEN PERIOD when "window of opportunity" is available for potential interventions and for effective health outcomes ${ }^{14}$. A mini review on thyroid gland anatomy, embryology and physiology defines both anatomical and physiological factors operating during embryonic stages of development of thyroid gland. Major attributable factors include abnormalities in ontogenesis of thyroid functions and impact of iodine concentrations imbalances. Based on the nature of the origin of disease process thyroid gland disorders may be dysgenetic due to anatomic defects or dyshormonogenetic due to impaired physiological maturation, synthesis and functioning of thyroid hormones .Thyroid gland is usually visible by embryonic day (E) E20 to E22 when "specification" of endodermal epithelium cells form thyroid anlage ${ }^{15}$.Normal embryology evolves in its first stage around two processes which are fetal thyroid gland development and maturation with evolution of the hypothalamic-pituitary-thyroid axis and any defect in this process results in thyroid agenesis. As a second stage of early morphogenesis thyroid anlage forms a bud that proliferates and migrates and then becomes bilobed around day E48 to E50. Any error or faulty lobulation results in hemi agenesis while an impaired descent causes an ectopic type of thyroid tissue ${ }^{15-17}$. The physiological component of thyroid hormones secretions begins around 10-11 weeks of age when thyroid peroxidase thyrotropin receptors initiate their production. The maturity of gland occurs in and around 25 weeks of gestation as is evidenced by a progressive rise in the ratio of free thyroxine (FT4) to thyrotropin $(\mathrm{TSH})^{16}$. At this stage defects in protein synthesis will result in dyshormonogenesis a disorder of the thyroid gland resulting in a deranged maturation synthesis or disorderly functioning of gland resulting in impaired thyroid hormones synthesis ${ }^{16,17}$.

Global status: According to WHO and International Council for the Control of Iodine Deficiency Disorders (ICCIDD) worldwide data on iodine status, regional differences in incidence of congenital hypothyroidism are suggested to be more likely due to iodine deficiency thyroid disorders rather than ethnic affiliation ${ }^{1,2}$. Iodine deficiency is the main cause of goiter in all ages. Across the globe, research studies on etiopathological factors of primary congenital hypothyroidism ( $\mathrm{PCH}$ ) report that $80 \%$ to $85 \%$ are due to thyroid agenesis , dysgenesis and ectopic thyroid while remaining $15 \%$ to $20 \%$ are due to dyshormonogenesis as a result of defective enzymatic deiodination metabolic disorders within the thyroid gland $^{7-9}$.In contrast to several previous results, a study from Turkey ${ }^{18}$ has reported thyroid dysgenesis in $52.2 \%$ and dyshormonogenesis in $47.8 \%$ of the study population. Similar results were observed in another study by Tamam et $\mathrm{al}^{19}$ in which dyshormonogenesis was etiology in $44 \%$ of subjects with permanent primary congenital hypothyroidism. Multiple factors which include mainly familial, maternal, neonatal and environmental factors seem to have combined etiological roles in occurrence of $\mathrm{CH}$.

Current status in India: Recent Indian studies report both higher occurrence of thyroid gland dysfunction in adults $^{13}$ and a parallel increase in prevalence of congenital hypothyroidism $(\mathrm{CH})$ in newborns ${ }^{10,11}$. Dyshormonogenetic goiters believed to be due to genetically determined 
iodine handling disorders cause various enzymes defects, impaired biosynthesis of thyroid hormones and result in congenital hypothyroidism. As per ICMR Task Force study on an epidemiological survey on endemic goiter, iodine deficiency is endemic throughout India in all states ${ }^{20}$. Predisposing factors include soil erosions, flooding, landslides and glaciers melting. Socio epidemiological data released by National Family Health Statistics ${ }^{21,22}$ have also documented that iodine imbalances are on the rise. As synthesis of thyroid hormones is fully dependent on iodine, such imbalances may trigger defective metabolic reactions and cause significant impact on health status of affected individuals. With high number of live births in India the most vulnerable newborns health needs to be addressed with priority. If not intervened appropriately impact on health may further pave way for pregnancy and perinatal complications 23,24 . Several research studies conducted in different parts of the country have reported both deficiency and excess of maternal urinary iodine. Results may be much more alarming in terms of prevalence of congenital hypothyroidism and warrants early detection and effective preventive strategies.

\section{Discussion}

Multiple factors which include mainly genetic and environmental factors play major roles in etiopathogenesis of $\mathrm{CH}$. Although India is classified as a country with adequate iodine intake based on the median UIC 249 million people including 8 million newborns annually are still unprotected from the lifelong consequences of IDDs ${ }^{13,16}$. The roles of type of salt used whether it is crystal or powder form, method of cooking and type of food frequently taken which could include goitrogens needs to be taken into consideration ${ }^{1,13}$. Impaired and inadequate iodine consumption can be attributed to the continuing diet choices and preference for sea salt or kosher salt instead of table salt which is a primary source of iodine. The practice of using crude crystal salt by the older generations due to belief that it contains more salty taste and also consumption of lesser quantity was an important observation recorded during study project tenure in South India. This warrants regional socio demographic risk factors analysis which may help to unfold etiological links for the much more higher prevalence of $\mathrm{CH}$ in South India born neonates. The increasing social culture and impact of using rock salt and organic salt with absent to varying concentrations of fortified iodine may be linked and warrant focused research studies for further correlations These observations further stress the absolute need of a monitoring system to assess and sustain adequate iodine content in consumed salt and focus on preventive strategies.

\section{Etiopathogenesis of Congenital Hypothyroidism}

The underlying causes of thyroid dysgenesis which includes thyroid agenesis, hypoplasia and ectopic thyroid remains obscure with only $2 \%$ to $5 \%$ having identifiable genetic mutations ${ }^{8}$. Currently Iodine Handling Disorders with iodine imbalances appear to be major factors in etiopathogenesis of $\mathrm{CH}^{14,15}$. It has been reported that in iodine deficient neonates turnover rate of intra thyroidal iodine is markedly accelerated and thyroid failure is more likely to occur.Thus a combined etiological role for both genetic and environmental factors seem to contribute for the occurrence of dyshormonogenesis as a major cause of primary $\mathrm{CH}$ in the entire Indian country in striking contrast with Western data where thyroid dysgenesis is more common $^{11}$.

Aflow chart with protocol for screening and confirmation of $\mathrm{CH}$ and key factors operating in etiopathogenesis are shown below. Four levels of operations are essential for evaluation of a neonate for $\mathrm{CH}$ after screening and confirmation of diagnosis of $\mathrm{CH}$.

Salient features of the flow chart are the following.

Key Risk Factors A - predisposing risks are predominantly seen in Indian population and cause physiological derangements of the thyroid gland e.g. - India and other Asian countries.

Key Risk Factors B - includes mainly genetic anatomical factors - e.g. USA, UK.

Key Risk Factors C- Multifactorial includes combination of factors - e.g. South Asia, Africa.

Thyroid dyshormonogenesis is emerging as a significant cause of congenital hypothyroidism $(\mathrm{CH})$ particularly in Asian population and is more frequently seen in India amongst southern states ${ }^{12}$.Indian studies report both higher occurrence of thyroid gland dysfunction in families ${ }^{21}$ and a parallel increase in prevalence of congenital hypothyroidism $(\mathrm{CH})$ in newborns ${ }^{12}$ With specific reference to the State Of Tamil Nadu a district wise survey conducted in 2013 by Dr Pandey ${ }^{1}$ has documented reduced household consumption of iodized salt at $18.2 \%$ which is inadequate as per WHO criteria An observation of similar data stress the need for extensive research studies among most vulnerable maternal, fetal, neonatal, infancy and under five age group populations. Dyshormonogenetic goiters believed to be due to genetically determined iodine handling disorders cause various enzymes defects impair biosynthesis of thyroid hormones and result in congenital hypothyroidism. Recent weighted estimate pooled from subnational surveys indicated that the median UIC of the population was $154 \mathrm{mg} \mathrm{L}-1$ and that $34 \%$ of Indians had UIC $<100 \mathrm{mg} \mathrm{L}-1$, indicating insufficient iodine intake ${ }^{1,8}$. Hence the role of iodine as a major causative factor needs to be further explored. Assessment of current iodine status in pregnant women is most essential and quantifications of urinary iodine concentrations and daily intake of iodine 
Protocol For Follow up Of Newborns with Congenital Hypothyroidism (CH) STEP 1 Screening by DBS [ DELFIA ]

STEP 2 Confirmation with serum TSH and FT4 [ Chemiluminescence ]
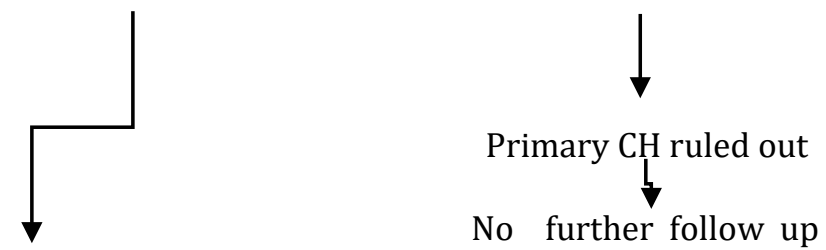

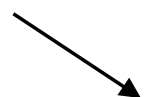

$\mathrm{R} / \mathrm{O}$ Central $\mathrm{CH}$

Primary CH confirmed

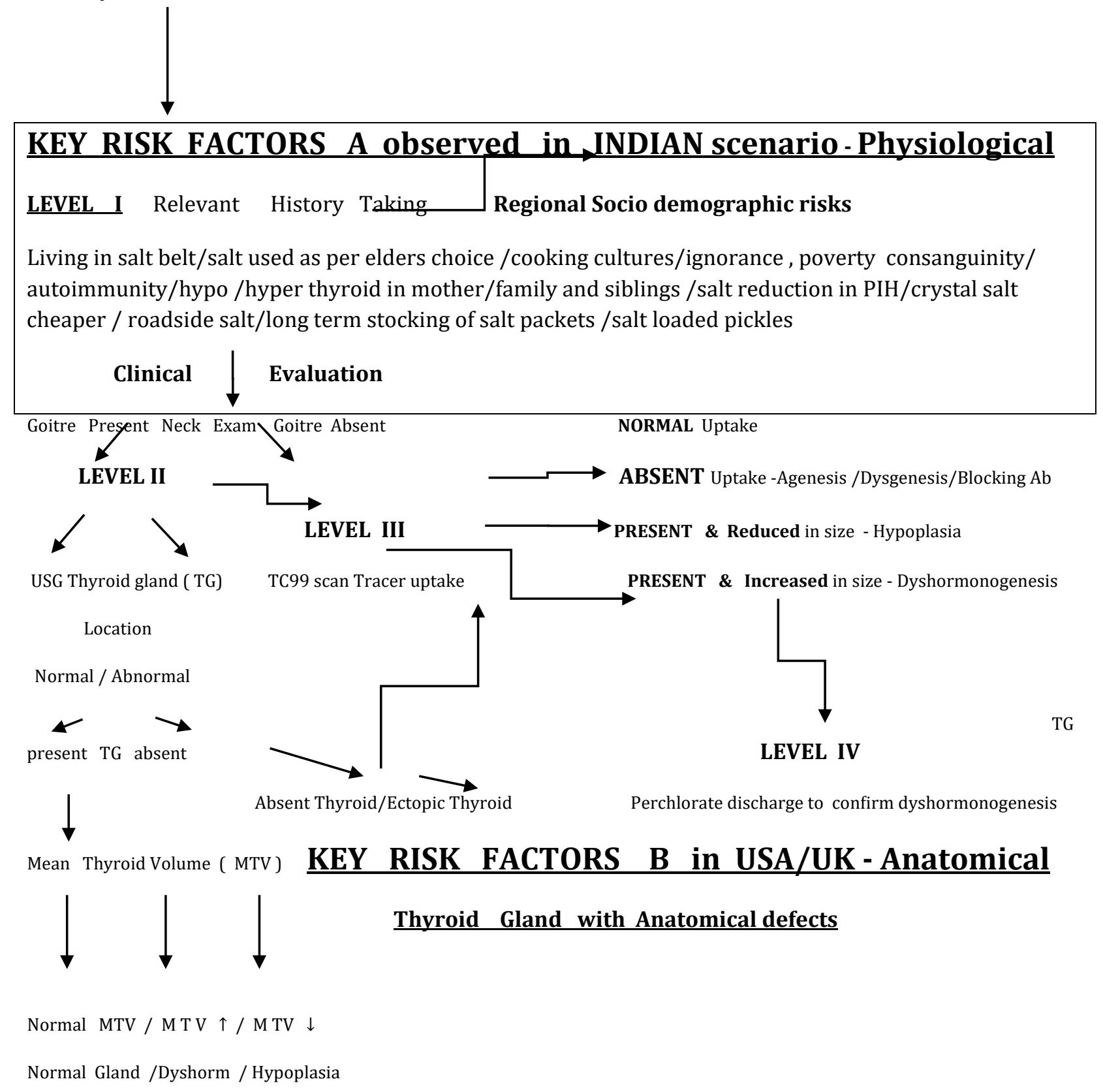

KEY RISK FACTORS C - Multifactorial SouthAsia/Africa MIXED FACTORS 
in salt will serve as basic data to study impact on thyroid glands functioning status and further evaluation with newborn spots screening for $\mathrm{CH}$ will serve to improve maternal and child health outcomes ${ }^{20}$.

Focus on ICMR data analysis in South India: Indian Council Of Medical Research (ICMR) conducted a pilot study on New Born Screening for $\mathrm{CH}$ and $\mathrm{CAH}$ during the period 2007 to 2012 in five selected states at Delhi, Chennai, Mumbai, Hyderabad and Kolkata with a target set to screen 1 lakh /one hundred thousand neonates .Project reports have been published recently and alarmingly pan ethnic prevalence of $\mathrm{CH}$ is reported as " 1 in 1172". Chennai centre has a higher prevalence of $\mathrm{CH}$ at " 1 in 727" respectively10,11 Worldwide several case reports have been published to support familial dyshormonogenesis ${ }^{12,24-27}$. Among ICMR project screened newborns the commonest cause of congenital hypothyroidism was dyshormonogenesis (47\%) followed by agenesis (20\%) and ectopia in $12 \%$ cases. In $21 \%$ cases the cause could not be clearly defined. In this pilot study at Chennai centre $90 \%$ were constituted by State of Tamil Nadu population and remaining $10 \%$ were represented by Andhra Pradesh state ${ }^{10-12}$. Out of a total of 20,517 neonates, 37 were confirmed to have $\mathrm{CH}$. 9 were born to consanguineous parents. 12 neonates had family history of hypothyroidism and 18 neonates had both parents with same nativity. A detailed clinical evaluation by a trained Paediatrician has documented a significant observation among $6 \mathrm{CH}$ confirmed neonates in that external congenital goiter was present in the neck. Ultrasound of thyroid showed an enlarged thyroid gland in normal position. Radiographs of knees showed both absent epiphyses and delayed epiphyseal ossification. Technetium 99 scan confirmed increased tracer uptake by an enlarged thyroid gland. Perchlorate Iodine I 131 discharge test confirmed diagnosis of dyshormonogenesis in 1 girl child. Costs, tertiary care access and social acceptance were significant obstacles to perform Perchlorate I 131 discharge test and plan genetic analysis in all suspected newborns. Though many specific point mutations are reported ${ }^{22,23}$ sensitive molecular studies from different populations with consistent phenotype genotype correlations are required to generalize DH patterns and prove etiologies. In pilot ICMR study ${ }^{10-12}$ among 37 neonates detected with $\mathrm{CH}$, 12 had a strong family history of thyroid disorders in that 5 mothers, 5 grandmothers and 2 fathers were affected. The presence of congenital goiter in 8 newborns and 18 parents belonging to the same geographical regions seems to further support the dominant role of dyshormonogenesis as causative factors. An increased risk of $\mathrm{CH}$ among infants with family history of thyroid diseases among parents has been reported in a clinical study done by Meddeet et al. ${ }^{24}$ in which three neonates detected with transient $\mathrm{CH}$ had their mothers having goiters with positive history of hypothyroidism has similarity to our data from ICMR pilot study on $\mathrm{CH}$ in India at South Regional centre ${ }^{10-12}$. The frequency of permanent $\mathrm{CH}$ infants having parents with hypothyroidism and goiter is higher and specifically with father reported to have thyroid disease. All these observations are strongly suggestive of multi-factorial origin of $\mathrm{CH}$ and emphazise the need for more clinical follow up data worldwide to unravel the most common causes of this preventable and treatable endocrine metabolic disorder.

\section{Conclusion}

With increasing prevalence of congenital hypothyroidism worldwide, evaluation of etiopathological links is crucial to provide timely therapy to prevent mental impairment and with more number of countries being declared iodine deficient from a sufficient status the role of iodine is unravelling and dyshormonogenesis emerging as a commoner cause of congenital hypothyroidism is clearly visible warranting stringent therapeutic and preventive strategies.

\section{Acknowledgements}

1. ICMR Task Force on Inherited Metabolic Disorders. NBS and HRS on CH and CAH , Members of the ICMR Task Force. AIIMS, New Delhi, India.

2. Medical and paramedical faculties at Institute of Obstetrics and Gynaecology Hospital for Women \& Institute of Child Health and Hospital for Children, Egmore Chennai, India.

3. Dr. Shriraam Mahadevan Professor, Department of Endocrinology , Sri Ramachandra Medical College, Chennai, Tamil Nadu, India.

4. Fetal Care Research Foundation, Chennai, Tamil Nadu, India.

\section{Conflict of interest}

None

\section{Funding}

None

\section{References}

1. Pandav CS, Yadav K, Srivastava R. et al. Iodine deficiency disorders (IDD) control in India. The Indian Journal of Medical Research. 2013; 138(3): 418-433.

2. WHO, UNICEF \& International Council for the Control of Iodine Deficiency Disorders. Global Prevalence of Iodine Deficiency Disorders (WHO, Geneva, 1993)

3. Geneva: World Health Organization; 2007. ICCIDD, UNICEF, WHO. Assessment of iodine deficiency disorders and monitoring their elimination: a guide for programme managers.

4. Kaur G, Anand T, Bhatnagar N, et al. Past, present, and future of iodine deficiency disorders in India: Need to look outside the blinkers. J Family Med Prim Care. 2017; 6: 182-90 
5. Nair PS, Sobhakumar S, Kailas L. Diagnostic re-evaluation of children with congenital hypothyroidism. Indian Pediatr. 2010; 47: 757-60.

6. Jacob JJ. Neonatal Screening for Congenital Hypothyroidism with Focus on Developing an Indian Screening Programme. European Endocrinology. 2016; 12(2): 99-103.

7. Caldwell KL, Makhmudov A, Ely E, et al. Iodine status of the U.S. population, National Health and Nutrition Examination Survey, 2005-2006 and 2007-2008. Thyroid. 2011; 21: 41927.

8. Andersson M, Karumbunathan V, Zimmermann MB. Global iodine status in 2011 and trends over the past decade. J Nutr. 2012; 142: 744-50.

9. Hannoush ZC, Weiss RE. Defects of Thyroid Hormone Synthesis and Action. Endocrinology and metabolism clinics of North America. 2017; 46(2): 375-388.

10. ICMR Task Force on Inherited Metabolic Disorders Indian J Pediatr. 2018. https://doi.org/10.1007/s12098-017-2550-7.

11. ICMR Task Force on Inherited Metabolic Disorders Indian J Pediatr. 2018. https://doi.org/10.1007/s12098-018-2645-9

12. Prabhu SR, Mahadevan S. Congenital Hypothyroidism Due to Dyshormonogenesis in 2 Siblings. Indian journal of pediatrics. 2018 Feb 15.

13. Lane, R.H. Fetal programming, epigenetics, and adult onset disease. Clin. Perinatol. 2014; 41: 815-831.

14. Walker SP, Wachs TD, Gardner JM, et al. International Child Development Steering Group. Child development: risk factors for adverse outcomes in developing countries. Lancet. 2007; 369(9556): 145357.

15. Obregon MJ, Calvo RM, del Rey FE, de Escobar GM. Ontogenesis of Thyroid Function and Interactions with Maternal Function. Endocr Dev. 2007; 10: 86-98.

16. Patel J, Landers $\mathrm{K}, \mathrm{Li} \mathrm{H}$, et al. Thyroid hormones and fetal neurological development. Journal of Endocrinology, 2011; 209:

17. Nilsson M, Fagman H. Mechanisms of thyroid development anddysgenesis: an analysis based on developmental stages and concurrent embryonic anatomy. Curr Top Dev Biol. 2013; 106: $123-170$

18. Bezen D, Dilek E, Torun N, et al. Etiological evaluation of primary congenital hypothyroidism cases. Turkish Archives of Pediatrics/Türk Pediatri Arşivi. 2017 Jun; 52(2): 85.

19. Tamam M, Adalet I, Bakir B, et al. Diagnostic spectrum of congenital hypothyroidism in Turkish children. Pediatr Int. 2009; 51: 464-8.

20. An ICMR Task Force Study. New Delhi: ICMR; 1989. Indian Council of Medical Research (ICMR). Epidemiological survey of endemic goitre and endemic cretinism.

21. National Family Health Survey (NFHS-2), 1998-99: India. I. Mumbai: IIPS; 1999. International Institute for Population Sciences (IIPS), Macro International.

22. National Family Health Survey (NFHS-3), 2005-06: India. I. Mumbai: IIPS; International Institute for Population Sciences (IIPS) and Macro International. 2007.

23. Zimmermann MB. The effects of iodine deficiency in pregnancy and infancy. Paediatric and perinatal epidemiology. 2012 Jul 1; 26(s1): 108-17.

24. Medda E, Olivieri A, Stazi Ma, et al. Risk factors for congenital hypothyroidism: results of a population case-control study (1997-2003). European journal of endocrinology. 2005; 153(6): 765-73.

25. Sparling DP, Fabian K, Harik L, et al. Fennoy Congenital hypothyroidism and thyroid dyshormonogenesis: a case report of siblings with a newly identified mutation in thyroperoxidase. Journal of Pediatric Endocrinology and Metabolism. 2016 May 1; 29(5): 627-31.

26. Ramesh BG, Bhargav PR, Rajesh BG, et al. Genotype-phenotype correlations of dyshormonogenetic goiter in children and adolescents from South India. Indian J Endocr Metab. 2016; 20: 816-24.

27. Castanet M, Lyonnet S, Bonaïti-Pellié C, et al. Familial forms of thyroid dysgenesis among infants with congenital hypothyroidism. New England Journal of Medicine. 2000 Aug $10 ; 343(6): 441-2$. 